\title{
Gardner syndrome
}

\author{
SADJ July 2021, Vol. 76 No. 6 p378
}

\section{S Rajbaran Singh', Al Black ${ }^{2}$}

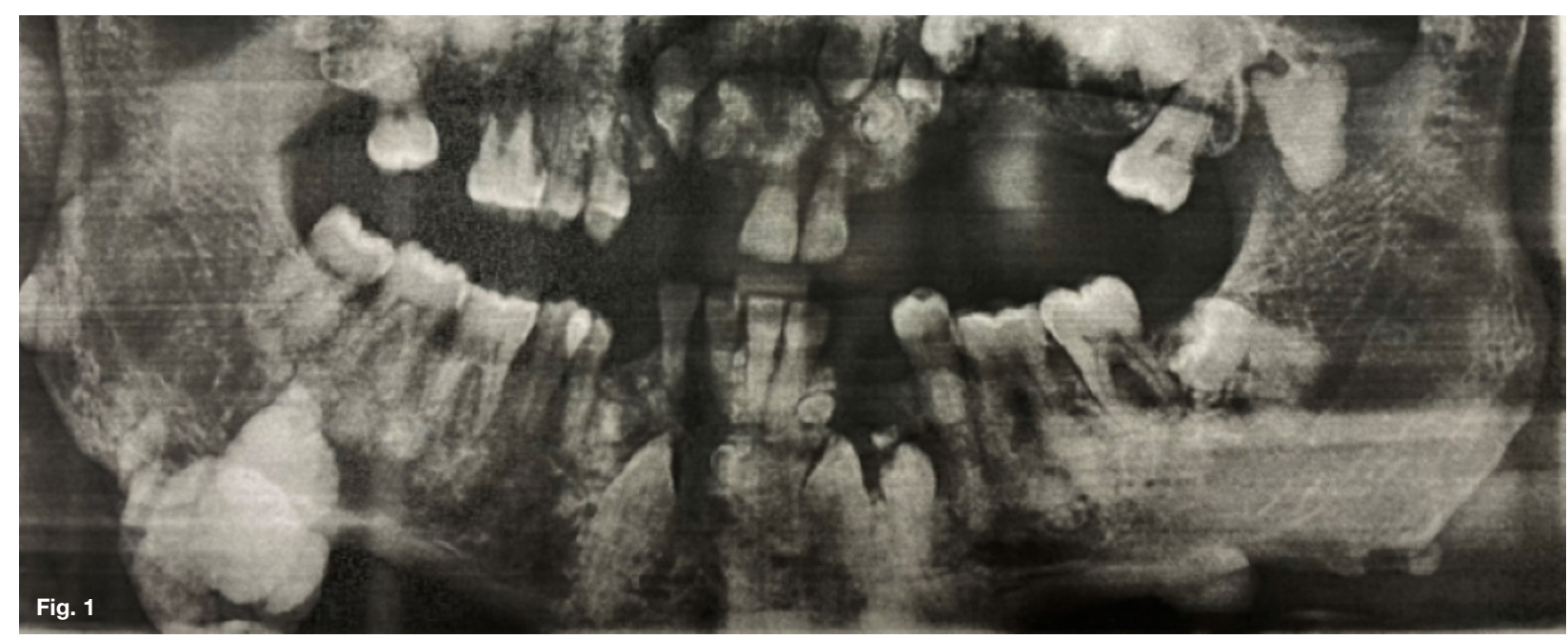

\section{INTERPRETATION}

A 58-year-old male patient was referred for a panoramic radiograph after having presented with a hard swelling of the right mandible. Panoramic radiographic examination (Fig. 1) demonstrated multiples dental anomalies and variable bone densities in both jaws prompting clinicians to "dig" further. Teeth 17, 12, 11, 26 and 27 were missing. Teeth 13, 23, 24, 25, 38, 34, 33 and 43 were unerupted. An unerupted supernumerary left mandibular molar appeared in the region of the mandibular coronoid process. Multiple, small, well-defined radiopacities of density comparable to odontogenic material were evident in the anterior maxilla and mandible. These opacities often demonstrated radiolucent borders consistent with the finding of multiple odontomas. Both jaws demonstrated variable bone density. Cottonwool like opacities partially blending into adjacent trabeculae were evident in the mandible and maxilla posteriorly. The contour of the inferior border of the mandible bilaterally in proximity to the angle was irregular with multiple, well-defined, smooth, lobulated homogenous radiopacities suggestive of osteomas. The radiographic features of multiple osteomas, odontomas, variable bone density, supernumerary and unerupted teeth warranted the referral of the patient for gastroenterological investigation to exclude Gardner Syndrome.

\section{Author affiliations:}

1. Sandeepa Rajbaran Singh: MSc., Dip.Odont, BChD, Dip.HRM, School of Oral Health Sciences, Sefako Makgatho Health Sciences University, Ga-Rankuwa, South Africa. ORCID Number: 0000-0002-7313-1365

2. Alan I Black: BSc, BDS, School of Oral Health Sciences, Sefako Makgatho Health Sciences University, Ga-Rankuwa, South Africa. ORCID Number: 0000-0003-0658-2542

Corresponding author: Sandeepa Rajbaran Singh

Department of Maxillofacial and Oral Radiology. School of Oral

Health Sciences, Sefako Makgatho Health Sciences University, Ga-

Rankuwa Unit 2, Ga-Rankuwa, South Africa.

Email: sandeepa.singh@smu.ac.za

Author contributions:

1. Sandeepa Rajbaran Singh: Primary author - $50 \%$

2. Alan I Black: Second author $-50 \%$
Gardner syndrome (GS), described as a variant of Familial adenomatous polyposis (FAP), comprises a classic triad of colonic polyps, multiple osteomas and an array of soft tissue tumours. A mutation of the Adenomatous polyposis coli (APC) tumour suppressor gene results in GS. Reported to have an incidence ranging between 1 in 8,000 and 1 in 14000 live births, GS bears no ethnic nor sex predilections and presents with a variety of colonic and extra-colonic manifestations. ${ }^{2}$ GS may present between 2 to 70 years of age and polyp formation carries a $100 \%$ risk of malignant transformation by the fourth decade. ${ }^{3,4}$ Bone manifestations precede intestinal polyps with osteomas presenting in up to $82 \%$ of patients. The maxilla and mandible are most often affected by osteomas with the frontal bone being the most common site for osteomas of the skull. The angle of the mandible commonly demonstrates lobulated osteomas that arise from the mandibular cortex. ${ }^{5}$ Most GS patients demonstrate a variety of dental anomalies that include unerupted teeth, congenitally missing teeth, supernumerary teeth, altered root morphology and odontomas. Recognition of the extra-colonic manifestations on panoramic examination facilitates the timeous identification of patients with GS and prompt referral for management.

\section{References}

1. Bisgaard ML, Fenger K, Bulow S, Niebuhr E, Mohr J. Familial adenomatous polyposis (FAP): frequency, penetrance and mutation rate. Hum Mutat. 1994; 3: 121-5.

2. Fotiadis C, Tsekouras DK, Antonakis P, Sfiniadakis J, Genetzakis M, Zografos GC. Gardner's syndrome: A case report and review of the literature. World J Gastroenterol. 2005; 11(34): 5408-11.

3. Butler J, Healy C, Toner M, Flint S. Gardner syndrome- review and report of a case. Oral Oncol Extra. 2005; 41: 89-92.

4. Cankaya AB, Erdem MA, Isler SC et al. Oral and Maxillofacial Considerations in Gardner's Syndrome. 2012; 9(2): 137-41. 